\title{
Comunicación grupal en Whatsapp para el aprendizaje colaborativo en la coyuntura COVID-19
}

\section{Group comunication on Whatsapp for collaborative learning in the covid-19 juncture}

\author{
Denis José Montenegro Díaz ${ }^{1}$ \\ https://orcid.org/0000-0001-7448-8051 \\ Universidad César Vallejo, Perú
}

Recibido: 10-06-2020

Aceptado: 13-08-2020

\section{Cita Recomendada}

Montenegro, D. (2020). Comunicación Grupal en Whatsapp para el aprendizaje colaborativo en la coyuntura COVID-19. Hamut'ay, 7 (2), 34-45.

http://dx.doi.org/10.21503/hamu.v7i2.2131

\section{RESUMEN}

En medio del estado sui-generis que vive el mundo con la pandemia del coronavirus (COVID-19), los grupos de conversación en WhatsApp han adquirido importancia significativa en el campo de la educación superior universitaria con un impulso notable al desarrollo del aprendizaje colaborativo entre estudiantes y docentes para el intercambio de mensajes educativos compartidos y contenidos de interés común de una clase. Al constituirse, se establecen interacciones comunicativas grupales, orientadas al desarrollo coordinado de diferentes actividades académicas, a través de la retroalimentación de mensajes entre sus participantes, que se registran desde el intercambio de experiencias, valores y prácticas cotidianas que dan lugar al aprendizaje colaborativo y encuentran fundamento en las teorías de usos y gratificaciones y de la complementariedad del canal. El objetivo del presente artículo ha sido revisar y contrastar investigaciones de los últimos cinco años, incluidos estudios de reciente aparición en la coyuntura del COVID-19, que describen las interacciones y contextos de uso de WhatsApp en la comunicación grupal para el aprendizaje colaborativo en educación superior universitaria entre estudiantes y docentes, mediante el empleo del análisis documental como técnica de investigación, basada en la búsqueda y revisión bibliográfica, contrastación informativa y organización de datos como parte del desarrollo metodológico y la perspectiva constructivista del conocimiento.

Palabras clave: WhatsApp, aprendizaje colaborativo, comunicación grupal, tecnologías, COVID-19.

\section{Abstract}

In the midst of the sui-generis state that lives the world with the coronavirus pandemic (COVID-19), conversation groups on WhatsApp have gained significant importance in the field of university higher education, with a remarkable boost to the development of collaborative learning between

\footnotetext{
1 Docente investigad $o r$ en la Universidad César Vallejo, Licenciado en Ciencias de la Comunicación, egresado de la Universidad Particular de San Martín de Porres. Magister en Docencia Universitaria y Gestión educativa, Universidad Alas Peruanas.

djmontenegro1@gmail.com
} 
students and teachers for the exchange of shared educational messages and content of common interest of a class. Being formed, group communicative interactions are established, aimed at the coordinated development of different academic activities, through the feedback of messages among their participants that are recorded from the exchange of experiences, values and daily practices that give rise to collaborative learning and find foundations in the theories of uses and gratifications and the complementarity of the channel. The objective of this article has been to review and contrast research over the past five years, including studies of recent appearance at the juncture of COVID-19, which describe the interactions and contexts of WhatsApp use in group communication for collaborative learning in university higher education between students and teachers, through the use of documentary analysis as a research technique, based on bibliographic search and review, informational contrast and organization of data as part of methodological development and the constructive perspective of knowledge.

Keywords: WhatsApp, collaborative learning, group communication, technologies, COVID-19.

\section{INTRODUCCIÓN}

La comunicación grupal en WhatsApp, ha configurado prácticas diarias casi indispensables que, en los últimos años, involucraron nuestras actividades personales, familiares, amicales, laborales, estudio y ocio. Antes de la llegada del COVID-19, los intercambios comunicativos en el contexto educativo en grupos de WhatsApp, permitían establecer contactos e interacciones interpersonales de forma física y remota entre estudiantes y docentes para el ejercicio de diferentes actividades colaborativas en la construcción de aprendizajes grupales. En tiempos de la pandemia, estas interacciones colaborativas grupales por WhatsApp, se han limitado tan solo al contacto remoto entre las personas; principalmente, mediante el uso de dispositivos móviles ante la inamovilidad poblacional decretada en todos los países en el mundo. Este artículo presenta así, algunas experiencias educativas universitarias, desde la revisión de algunas investigaciones recientes, en torno al uso de WhatsApp y las conversaciones grupales, que dan lugar a un conjunto de interacciones particulares para el aprendizaje colaborativo entre estudiantes y docentes universitarios y contrastados para describir como este medio, puede incidir significativamente en tal sentido, en el marco de la teoría de usos y gratificaciones y de la complementariedad del canal.

Las investigaciones revisadas han sido clasificadas en tres grupos; (1) artículos referidos al uso grupal de WhatsApp en contextos educativos universitarios, que identifican procesos y características de los aprendizajes colaborativos evidenciados, que destacan Jain, Luaran y Rahman.(2016), Fuentes, et, al (2017), Gómez Del Castillo (2017), Hassan \& Ahmed, A. (2018), Urien, Erro-Garcés y Osca (2019), Kizel (2019), entre otros, y (2) artículos e informes institucionales publicados en el año 2020, que analizan la coyuntura educativa universitaria, en medio de la pandemia COVID-19 (D’Orville, 2020, Sanz, Sáinz, Capilla, 2020). Así mismo, subyace la revisión de algunos documentos orientados al análisis de WhatsApp, desde las perspectivas teóricas de los Usos y gratificaciones (Fondevila-Gascón, et, al, 2019), (Igartua, Ortega-Mohedano y Arcila-Calderón, 2020) y la teoría de la complementariedad del canal (Casero-Ripollés,2020). La relevancia de este estudio documental, enfatiza la importancia del aprendizaje colaborativo, desde las interacciones comunicativas generadas en el uso de WhatsApp en un contexto educativo universitario, compuesto por estudiantes y docentes.

\section{MÉTodo}

Se presenta un análisis teórico-conceptual de artículos científicos publicados en revistas indexadas, 
desde la técnica del análisis documental y la metodología propuesta por Girao-Goris, et, al. (2007), que describe las etapas en la elaboración de una revisión bibliográfica, mediante la definición de los objetivos, búsqueda de la literatura, organización de información y redacción del artículo. (Vera, 2009)

Se tratan de contenidos actuales, que implican la comunicación grupal por WhatsApp y el aprendizaje colaborativo desde la perspectiva coyuntural del COVID-19, para lo cual, se realizó una exploración inicial exhaustiva de fuentes de información, a través del uso de base de datos como Scielo, Elsevier, SpringerLink, ProQuest, Redalyc, Dialnet y Google Scholar, así como algunos repositorios institucionales de universidades de México, Argentina y España. Se consideró entre los descriptores utilizados, aquellos términos referidos a grupos de WhatsApp y aprendizaje colaborativo, ventajas y desventajas del uso educativo de WhatsApp, actividades colaborativas en WhatsApp en el proceso de enseñanza-aprendizaje en entornos universitarios y visión del contexto educativo universitario mundial durante la pandemia del COVID-19.

Para el análisis y revisión de la literatura se consideraron dos etapas de búsqueda. En primer lugar, la búsqueda de publicaciones comprendidas entre los años 2015 a 2019, como antecedentes para la contextualización del fenómeno de estudio e inferir sus resultados, mediante un conducto relacional con las publicaciones de reciente aparición. En una segunda etapa, se realizó la búsqueda de publicaciones del ańo 2020, con el fin de recabar datos provenientes de investigaciones de reciente aparición, en cuanto a la situación del contexto educativo universitario, en medio de la pandemia del COVID-19 y el uso actual que tiene WhatsApp, en entornos educativos virtuales para el aprendizaje colaborativo grupal. Como estudio documental, este artículo, aborda a su vez la problemática del uso de WhatsApp, desde el enfoque de la teoría de usos y gratificaciones, y del paradigma evolutivo de la teoría de la complementariedad del canal, a fin de distinguir los aportes y cambios comunicativos en el entorno educativo, registrados con las TIC.

\section{Uso de Whatsapp y Grupos de Comunicación}

En los últimos años, WhatsApp como sistema de mensajería instantánea (SMI), ha masificado la popularización mundial de uso entre las personas, fundamentalmente jóvenes, quienes invierten muchas horas al día, para establecer contacto comunicativo con sus amigos, pareja, familiares, compañeros de trabajo, etc. Se trata de SMI, que goza de una mayor adaptabilidad y funcionalidad en dispositivos móviles para el acceso e intercambio informativo entre contactos mediante mensajes registrados en tiempo real y permiten compartir contenidos multimedia como texto, imágenes, audio, video y datos de ubicación (Scribano, 2017, cit. Dayani Ahada \& Ariff Lim, 2014 p. 189)

En el ámbito educativo, el uso de WhatsApp implica la necesidad de un conocimiento mutuo del entorno virtual para el intercambio de mensajes, producido en emisores y receptores y su red de contactos que se pueden apreciar en la figura 1 (Gómez Del Castillo, 2017). Para Suarez (2018), el uso educativo de WhatsApp se remite a la creación ocasional de un grupo, formado por los estudiantes y que pueden incluir al docente. En este sentido, cabe precisar las necesidades e intereses con fines educativos que emergen para la conformación de un grupo de conversación en WhatsApp entre estudiantes.

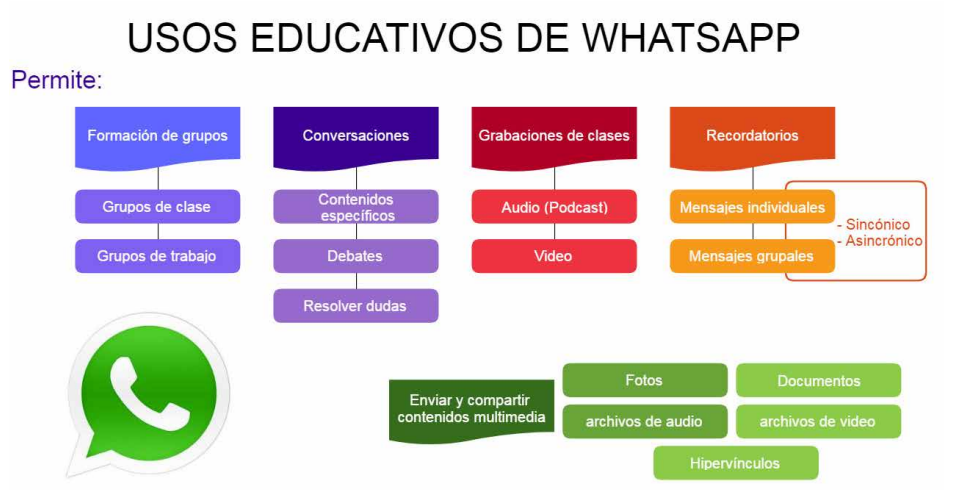

Figura 1: Usos educativos de WhatsApp Fuente: Suárez, 2018, Mosquera, 2016, García, 2014, Sanz, 2014

Elaboración propia (2020)

Por sus características, WhatsApp es capaz de propiciar mayores probabilidades para la producción de interacciones interpersonales desde la comunicación grupal, para el aprendizaje colaborativo en la producción de discursos comunicativos (Rubio y Perlado, 2015, p. 81) que favorecen el trabajo 
colaborativo grupal (Gómez Del Castillo, 2017, cit. Oliveira, Versuti \& Vaz, 2016), desde la practicidad de un smathphone. Su uso habitual, tiene un carácter fático y performativo, que posibilita formas de interacción comunicativa con fines referenciales y expresivos (López-Cantos, 2017). Para Fuentes et, al, (2017), la relación de dependencia entre el Smartphone y WhatsApp, parece estar fuertemente influenciada por el dinamismo de los grupos y aquellos surgidos desde el entorno de la clase (p.155), donde cada estudiante, asume un comportamiento de inclusión o exclusión en el debate e intercambio de mensajes, si percibe que estas relaciones comunicativas tienden a ejercer sentimientos de control, dependencia al medio, asunción de roles, empoderamiento, liderazgo de participación grupal, entre otros (p.167), e influencian en su forma de participar o interactuar.

Antes de la aparición del COVID-19, WhatsApp ya se empleaba como una herramienta de comunicación para reforzar las actividades colaborativas grupales entre estudiantes pertenecientes a una clase, con la presencia del docente como moderador. En las comunicaciones fuera de clase, Elhay \& Hershkovitz (2019), referencian a Aaen \& Dalsgaard 2016; Odewumi et al.2017, quienes consideran que WhatsApp facilita esta práctica como ninguna otra red social, al personalizar el uso del medio de acuerdo a las características y necesidades de cada estudiante, lo cual facilita la formación de grupos, que contribuye al desarrollo de sus oportunidades de aprendizaje. En esta perspectiva, la comunicación grupal, subyace a la luz del enfoque de estudios teóricos como los de Katz y Lazarfeld (1979), en donde la comunicación interpersonal, representa el primer eslabón para la conformación de redes comunicativas interpersonales y el establecimiento de grupos primarios que determinan las actitudes de cada miembro (Alsina, 2018, Saperas, 1992, p,36). Por su parte, Alsina (2018) cita a Visscher (1993, p. 472) quien manifiesta que la comunicación en cada grupo presentará características distintas que afectan sus modos de conversar, transmitir información, debatir o resolver conflictos para mantener o modificar relaciones afectivas.

Como núcleos de organización, los grupos de conversación en WhatsApp, han adquirido así, un rol de suma importancia en el desarrollo de estas prácticas discursivas diarias que fomentan el trabajo colaborativo para la construcción del aprendizaje, y que funcionan por diversos motivos como pequeñas comunidades (Lacanna, 2018, cit. Eckert y McConnell, 1992). Es así que, cada grupo, estará conformado por grupos de amigos, compañeros de trabajo o de estudio, grupos familiares, recreativos, etc, con características relativas al género, edad, intereses o vínculos que comparten sus participantes, para establecer relaciones estratégicas de comunicación, bajo un grado de indireccionalidad, cercanía, confianza, solidaridad y/o poder. (Lacanna, 2018)

En el contexto educativo universitario, algunas experiencias de comunicación grupal en WhatsApp, han permitido así que, la interacción entre sus usuarios, respondan a sus estilos de vida (Karapanos, et, al,2016), a partir del establecimiento de comunicaciones sincrónicas que caracterizan sus prácticas discursivas, en reemplazo de la llamada convencional (Rubio-Romero \& Perlado, 2015). En tal sentido, Fuentes et al. (2017) destacan las expresiones de Rambe \& Chipuza (2013), al ratificar que el uso de WhatsApp se encuentra inexorablemente ligado al del smartphone como dispositivo móvil, que permite a los estudiantes universitarios acceder a funciones como "compartir información" para asumir roles "académicos de asesoría» con sus demás compańeros en la colaboración grupal. Otras funciones comunicativas en WhatsApp, han sido identificadas por Montenegro \& Hermenegildo (2018) mediante el uso de emojis en estas conversaciones grupales, al propiciar un intercambio comunicativo de mensajes icónicos, con mayor fluidez, expresividad y menor formalidad a cada usuario, que atribuye un determinado valor o interpretación. Los gifs animados o stickers con imágenes personalizadas, creados con aplicaciones para Android como artificios comunicativos, resultan así mismo, formas dinámicas de interacción comunicativa registradas en estas conversaciones.

En contraparte, WhatsApp posee al mismo tiempo una serie de dificultades y limitaciones para sus usuarios. En primer lugar, por el problema de la flexibilidad lingüística que WhatsApp otorga a sus usuarios para comunicarse de manera libre, mediante mensajes textuales, iconográficos y multimedia, que hace que los intercambios comunica- 
tivos grupales, puedan tener repercusión negativa, por la inadecuada utilización ortográfica y gramatical del lenguaje (Kisel,2019). En segundo lugar, por el tiempo que actualmente los jóvenes estudiantes, invierten para establecer extensas conversaciones escritas en WhatsApp con fines concretos para el aprendizaje, que pueden redundar en consecuencias adversas en el rendimiento académico, referidas por Yeboah \& Ewur (2014) como argumentaciones (Vilches y Reche, 2019). Tercero, por afectar la privacidad de los usuarios, al exponer los números telefónicos de sus dispositivos móviles en la vista pública del grupo de conversación entre estudiantes y docentes (Tang \& Hew, 2020). Cuarto, por el problema de la desregulación en el uso del chat grupal, que tiende a sobresaturar de mensajes a los participantes y abrumarlos por su escasa claridad comunicativa y para la coordinación grupal hacia el desarrollo de actividades conjuntas (Vilela, Urbano y Díaz, 2015). En esta lid, Vilches y Reche (2019), distinguen dos grupos de limitaciones que posee WhatsApp en el marco de desarrollo de las actividades colaborativas universitarias; a) limitaciones en las relaciones interpersonales, que comprenden aquellos malentendidos, conflictos e inconvenientes suscitados entre los miembros de un grupo para asumir responsabilidades del trabajo grupal, y b) las limitaciones en el proceso de comunicación que se generan a causa de conversaciones extensas y las limitaciones para el uso y comprensión del lenguaje no verbal, que puedan registrar algunos participantes en el intercambio de mensajes. Para remediar estas situaciones, Man (2014, cit. Vilches y Reche, 2019) plantea algunas recomendaciones; (1) limitar el grupo a 20 miembros, (2) empleo de nombres reales y no alias de los integrantes en un grupo, (3) proveer por igual a todos los integrantes del grupo, los recursos que necesiten para desarrollar una competencia digital, y (4) crear reglas sobre uso del grupo. Un aspecto que no puede dejarse de lado, son los comportamientos derivados del uso recurrente de WhatsApp en estudiantes, recopilados en investigaciones previas por Lacanna (2018), que originan algunos desordenes como, trastornos de estrés o ansiedad, phubbing y snubbing (Capilla y Cubo, 2017), nomofobia, síndrome de la llamada imaginaria, doble clic y la dependencia generada hacia internet (Giménez y Zirpoli, 2015). Cabe destacar en esta lid, las prácticas de sexting desarrolladas por jóvenes y adultos, identificadas por López-Cantos, 2017, en referencia a las investigaciones de Drouin \& Ladgraff, 2012, Weisskirch \& Delevi, 2011). En suma, la comprensión e interpretación de cada mensaje en las conversaciones de grupo en WhatsApp, dependerá de las características que sus participantes desarrollen en cuestión de los fines, alcances e intereses de sus intercambios comunicativos hacia el logro del aprendizaje colaborativo, a partir de las ventajas y desventajas descritas del medio.

\section{Usos y Gratificaciones de Aprendizaje en What- sapp}

En el escenario educativo universitario mundial producido por el COVID-19, el uso de WhatsApp como herramienta para la comunicación grupal y el aprendizaje colaborativo, comparado al de otras redes sociales, queda fundamentada desde la concepción teórica de los usos y gratificaciones de Katz, Lundberg, Hulten, Mc Quail y otros, al postular la selección y elección de un medio de comunicación por parte del público, con la finalidad de obtener un consumo informativo para satisfacer sus necesidades e intereses particulares que promueven "gratificaciones".

Igartua, Ortega-Mohedano \& Arcila-Calderón (2020), sostienen que WhatsApp, ha tenido un uso prevalente en comparación de medios tradicionales y otras redes sociales, donde el consumo informativo público ha sido mayor desde el inicio de la pandemia del coronavirus. En España, un estudio de medios, determinó que en las cuatro semanas iniciales de inamovilidad social producida por la pandemia, el consumo informativo fue liderado por la televisión (86.2\%) y WhatsApp (77.6\%) por su frecuencia de uso (Moreno, Fuentes-Lara y Navarro, 2020); no obstante, también vinculado al aumento de la desinformación pública por noticias falsas o "Fake news", a causa del temor público de creer que los hechos informados por los medios de comunicación en torno a la pandemia, resultaban apócrifos en medio del confinamiento social (Sánchez-Duarte y Magallón Rosa,2020). En cuanto a otros países, no se lograron obtener referencias recientes similares acerca del consumo 
informativo de medios, que, en términos generales, ha encumbrado el acceso a la información pública a nivel mundial, desde el uso de redes sociales como WhatsApp.

En la misma dirección que postula los usos y gratificaciones, la teoría de la complementariedad del canal, se presenta como un paradigma reciente, que pretende evolucionar esta concepción, a partir de la irrupción de las nuevas tecnologías y el carácter de predictibilidad que tienen los medios de comunicación en la actualidad para la búsqueda de información (Moreno, Fuentes-Lara y Navarro, 2020, cit, Dimmick; Chen; Li, 2004). Así, las plataformas digitales, propician interacciones que complementan el proceso de recolección informativa, a partir de la mayor atención del ciudadano sobre el contenido, en lugar de prevalecer el uso específico de un medio para obtener información. (Casero-Ripollés, 2020, cit. Dutta-Bergman, 2004). Esto nos da una idea general de como la información transmitida por redes sociales durante el desarrollo de la crisis sanitaria mundial desatada por la pandemia, ha otorgado mayores usos y gratificaciones que los medios masivos tradicionales.

En circunscripción al ámbito educativo universitario, el uso de la mensajería instantánea móvil que pueden registrar los estudiantes, da lugar a cinco formas de gratificación, identificadas Huang y Leung (2009); (1) utilidad interpersonal, (2) utilidad social, (3) conveniencia, (4) información y (5) entretenimiento (Fondevila-Gascón, et, al, 2019). Por su parte, Jain, Luaran \& Rahman, (2016) identifican cinco beneficios generales hacia el aprendizaje desde el uso de WhatsApp como sistema de mensajería instantánea; (i) desarrolla la cooperación en línea entre docentes y alumnos, (ii) gratuidad y facilidad de uso, (iii) capacidad del mensaje instantáneo para segmentar el aprendizaje con facilidad, mediante comentarios, a través de una conversación relacionada al curso, (iv) permite generar una publicación de clase a los estudiantes, con la finalidad que otros estudiantes puedan comentarlo para fomentar la colaboración y (v) facilidad para la construcción de la información y el conocimiento.

Se considera así, que el uso de WhatsApp permite potenciar la comunicación grupal, como ente generador de la participación y formas de interacción de los estudiantes hacia el aprendizaje colaborativo, a través de acciones individuales y colectivas que permiten acopiar e intercambiar información; capaz de desarrollar habilidades potenciadoras del aprendizaje, que se manifiestan desde las valoraciones como actitudes y emociones favorables o desfavorables que presentan los estudiantes en la interacción grupal que redundan en gratificaciones. (Lizcano-Dallos, Barbosa-Chacón y Villamizar-Escobar, 2019, cit. Bennett, Bishop, Dalgarno, Waycott, Kennedy, 2012, Kozel, Klézl y Pawlasová,2013)

\section{El Aprendizaje Colaborativo en Grupos de Whatsapp}

Como componente del enfoque constructivista de la educación, el aprendizaje colaborativo tiene como fin supremo, propiciar las prácticas cooperativas de aprendizaje en un grupo social. Para Lizcano-Dallos, Barbosa-Chacón y Villamizar-Escobar (2019), se trata de un tipo de aprendizaje que facilita la interacción y aparición de vínculos afectivos y emocionales muy fuertes entre sus participantes, basado en el uso de las TIC y se relacionan a su vez con asociaciones discursivas que pasan desde las actividades de grupo, su intercambio informativo e interacciones de sus participantes, hasta las estrategias que puedan emplearse para la consecución de este fin.

Las prácticas discursivas en WhatsApp para el aprendizaje colaborativo, han sido materia de análisis de diferentes investigaciones en años anteriores, cuya importancia, radica en la eficacia de este intercambio comunicativo de mensajes. Para Castellanos y Onrubia (2015), analiza los procesos de regulación del aprendizaje desde el enfoque de la psicología educativa en tres enfoques teóricos; (1) la regulación metacognitiva, ligado al conocimiento que emerge en cada estudiante desde su propia cognición y su intencionalidad de aprendizaje; (2) el aprendizaje autorregulado, como resultado de la metacognición que conduce a la planificación, organización y realización de tareas, y (3) el aprendizaje colaborativo desde la regulación social que comprende el aspecto individual y grupal, en la manera como estas actividades mencionadas, 
tienden a ser monitoreadas y evaluadas, desde la participación y motivación de cada integrante. En tiempos del COVID-19, las universidades han tenido que adaptar sus formas de comunicación para lograr la colaboración en el aprendizaje, por medio de plataformas virtuales o sistemas de mensajería instantánea como WhatsApp, caracterizado por un fuerte intercambio informativo, que, a opinión de Franch y Camacho (2004, p. 227), implica mayor innovación y darse a conocer a todas las partes involucradas del mensaje. (Rivera-Rogel et al, 2019)

Esta percepción utilitaria de WhatsApp para la búsqueda de la eficacia del trabajo en equipo, es analizada por Urien, Erro-Garcés y Osca (2019), al incidir en los procesos cognitivos y hacia la toma de decisiones grupales, por medio del juego de roles. Esto atribuye, un potencial comunicativo favorable al desarrollo de actividades y actitudes colaborativas para al trabajo en equipo. En tal sentido, las conversaciones gestadas en grupos de WhatsApp, pueden constituir un factor clave en la construcción de aprendizajes colaborativos, que, según Kisel, (2019), surten efecto, como diálogos discursivos de los miembros de un grupo o comunidad de WhatsApp, con características únicas que identifica sus relaciones interpersonales, semejantes al lenguaje dialógico I-Thou (yo-tú) referido por Buber $(1970,2004)$, al describir que, las características discursivas existenciales de grupo se basan en relaciones interpersonales de mutua reciprocidad, franqueza y presencia de sus miembros. Desde esta perspectiva, la discusión de los participantes de un grupo de WhatsApp, se puede promover mediante temas de debate, que pueden ser consentidos y no consentidos por sus integrantes y distinguirá a sus relaciones interpersonales, comparadas a las desarrolladas por otros grupos.

Experiencias universitarias de aprendizaje colaborativo en grupos de WhatsApp, han sido también labor de varias investigaciones en estos últimos años, en diversos aspectos recopilados por Vilches y Reche (2019), como la adaptabilidad de las personas para el desarrollo de un trabajo colaborativo eficaz en un grupo de WhatsApp (Bouhnik \& Deshen, 2014), el acercamiento instructivo entre docentes y estudiantes en grupo de WhatsApp, como herramienta de colaboración de actividades grupales (Hidayanto \& Setyadi, 2014), su enri- quecimiento, a través del discurso audiovisual e icónico (Cremades, Maqueda y Onieva, 2016), y en torno a ello, para el acercamiento del lenguaje escrito a la oralidad con imágenes o gestos (Galán, 2011, Calero, 2014, Sampietro, 2016). Así mismo, la posibilidad de expandir el conocimiento, más allá de las aulas universitarias (Avci \& Adiguzel, 2017, Barhoumi, 2015, Ngaleka \& Uys, 2013, Rambe \& Bere, 2013), desde un intercambio rápido e instantáneo de mensajes (Morduchowicz, 2013), mediante la comunicación informal entre estudiantes (Fuentes et, al, 2017; cit. Cifuentes y Lents, 2011; Smit, 2012) y el contacto interpersonal sin distinción de tiempo o lugar. (Gómez Del Castillo, 2017, cit. Iniciarte, 2004)

En el ámbito metodológico, se destaca el aporte de Vilela, Urbano y Díaz (2015), en la elaboración de un instrumento de recolección de datos con escala tipo Likert, para describir las características de uso de WhatsApp en el trabajo colaborativo grupal. En el aspecto etnográfico, las conversaciones dialógicas en WhatsApp son analizadas por Vela y Cantamutto (2016), a través de la técnica de la observación participante, para la identificación de las características del análisis discursivo etnográfico de los individuos por medio de un Smartphone y en base a los diálogos bilaterales o multilaterales que gestiona un moderador.

De manera reciente, el análisis exploratorio de Bangasisi, et, al (2020), permite conocer cómo WhatsApp influye significativamente en la educación y el aprendizaje colaborativo, a través del rendimiento académico en la medición de sus procesos de aprendizaje colaborativo móvil y construcciones explicativas en estudiantes de la Universidad de Sudáfrica en diez dimensiones; (1) interacción, (2) soporte, (3) intercambio de información, (4)sentido de comunidad, (5) interdependencia, (6) confianza, (7) aprendizaje activo, (8) formalidad, (9) colaboración y (10) logro académico de construcción.

\section{La Experiencia Docente en Grupos de Whatsapp durante la Pandemia}

La pandemia del COVID-19, ha impuesto un riguroso reto educativo a los docentes; el ser más asertivos, resilientes y colaborativos con sus estudiantes. La experiencia docente indica que, ante 
una coyuntura mundial, sin precedentes parecidos en los últimos cien años, la educación necesita el apoyo de los recursos tecnológicos para desarrollarse. WhatsApp ha sido el medio que, por su practicidad de uso mediante dispositivos móviles, se ha adaptado con mayor versatilidad a las rutinas diarias de los estudiantes y docentes universitarios en esta nueva normalidad. Para Ruiz Larraguivel (2020), los docentes universitarios han debido modificar o reducir sus formas tradicionales de enseñanza y medios empleados en su proceder didáctico, para adaptarlos al medio virtual, desde las interacciones producidas en el entorno virtual, sin perder la efectividad de una clase presencial. Martínez, (2020), pone de manifiesto que, la mayor preocupación educativa en el confinamiento social impuesto por la pandemia, han sido las discusiones mediatizadas en redes sociales, en cuanto a la forma de cómo se manejarían las evaluaciones. Bajo esta incertidumbre social, un gran sector de la población mundial, tuvo opiniones desconfiadas acerca de la calidad educativa que podrían generarse con las clases remotas, que hace referencia D'Orville, (2020), en el sentido que el sistema educativo mundial, debió adaptarse pronto al uso de las tecnologías de información y comunicación, sistemas de mensajería instantánea y plataformas virtuales, a través de Internet.

Con ello, se buscó dar soluciones de apoyo al sistema educativo desde cada gobierno, a fin que las clases no se paralicen por la pandemia, desde una perspectiva de inclusión, equidad e igualdad de género, propuesta por la Coalición Mundial por la Educación de la UNESCO. Como parte de este análisis, Hodges et al, (2020) intenta esclarecer diferencias conceptuales entre enseñanza remota de emergencia y el aprendizaje en línea. En el primer caso, cita a Peixoto, Correia \& Rego (2020) al destacar "el intercambio concertado de conocimientos y experiencias" desde la labor docente, lo que conduce a que adquiera un rol cada vez más protagónico en el control e implementación del diseño de su curso, mediante el apoyo logístico brindado por la universidad. En el caso del aprendizaje en línea, por medio de una adecuada planificación que coadyuve a identificar aquellos contenidos a ser cubiertos con los estudiantes, bajo la observación de las interacciones registradas en desarrollo del proceso de aprendizaje. (Hodges, et, al, 2020, cit. Bernard, et al., 2009). La pandemia ha impuesto así, una ardua exigencia para las universidades, al brindar capacitación a sus docentes para el manejo de estos entornos virtuales, en medio de la coyuntura sanitaria, que caracteriza el proceso de enseñanza-aprendizaje, desde la construcción de conocimientos basado en la colaboración grupal. El rol del docente universitario como actual administrador de la educación superior en el entorno virtual, trasciende también, en la toma de decisiones que con los estudiantes, se toma a corto, largo y mediano plazo. (Sánchez Mendiola, et al, 2020), con la presencia de un conjunto de dificultades identificadas desde la perspectiva docente, de acuerdo a lo apreciado en la figura 2, desde una experiencia registrada en la UNAM, México.

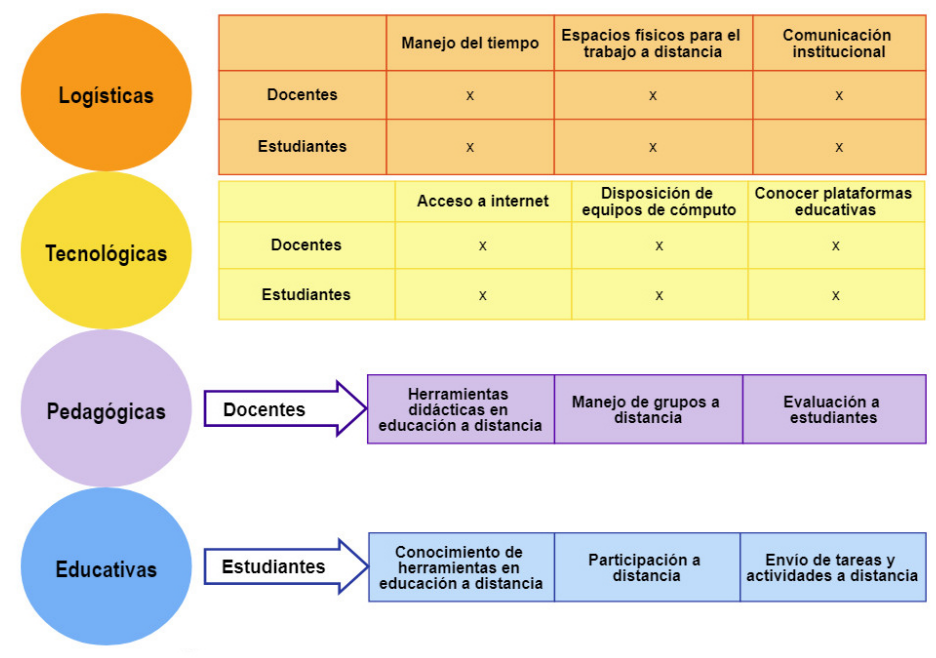

Figura 2: Problemáticas en docentes y estudiantes en educación virtual. Visión desde la perspectiva docente de la UNAM, México

Fuente: Adaptado de Sánchez Mendiola, et, al, 2020. Elaboración propia

La figura 2, nos muestra las problemáticas que han sido identificadas por los mismos docentes de la UNAM, acerca de cómo estudiantes y docentes han tenido que afrontar con responsabilidad, el proceso de enseñanza-aprendizaje en medio de la pandemia y sus relaciones personales, familiares y académicas, ligadas a los aspectos tecnológicos, socioafectivos o logísticos, de acuerdo a lo señalado por Sanz (cit Sánchez Mendiola, et,al, 2020), que ha generado un sin número de situaciones complejas, que requieren ser atendidas en el corto, 
mediano y largo plazo, a fin que los estudiantes universitarios, no vean afectado su aprendizaje y formación profesional con un impacto negativo.

\section{El Acompańamiento Pedagógico en las Activi- dades Colaborativas por Whatsapp}

El aprendizaje colaborativo en grupos de WhatsApp, no puede encontrarse ajeno a la supervisión docente; más aún, en tiempos del COVID-19, señalan así que, el aseguramiento de su efectividad, dependerá de factores como la participación del docente en línea para la enseñanza de los contenidos de un curso, las interacciones generadas en el aula virtual con sus estudiantes, la evaluación continua de los conocimientos y en menor cuantía cualitativa de la plataforma digital. Sanz, Saiz y Capilla (2020), citan a Sisko Mälinen, catedrática de la Universidad de Tampere en Finlandia, quien recomienda la participación sincrónica o asincrónica desde la interacción en directo del estudiante en una clase remota $\mathrm{o}$, a través de las descargas de contenidos educativos desde una plataforma virtual. Para Hassan \& Ahmed, (2018), WhatsApp puede coadyuvar a mejorar la motivación de los estudiantes, para brindarles un apoyo tutorial que permita mejorar sus calificaciones; cuyas conclusiones, han sido el resultado de una investigación con estudiantes de especialización de pregrado en cursos de inglés de la Universidad King Khalid en Arabia Saudita, para identificar sus interacciones con el docente de curso, a través de un grupo de WhatsApp. Suárez Lantaron (2017), refiere a García Antelo (2010), en manifiesto a la importancia de la tutoría como apoyo en las labores de acompañamiento docente al estudiante, desde un sentido formativo y orientador individual o grupal. Para Díaz, Iglesias y Valdés (2020), los medios digitales que brindan apoyo a la tutoría educativa, deben favorecer efectivamente las prácticas interactivas del proceso de enseñanza-aprendizaje, a fin de lograr confianza en el estudiante y reciba orientación metodológica en su formación. En síntesis, la nueva normalidad impuesta por la pandemia, exige mayor compromiso y dedicación a que los docentes, incidan sus actividades como tutores, desde el monitoreo permanente de su trabajo, atención de consultas, verificación del progreso de sus estudiantes, e inculcarles un sentido de responsabilidad personal, que coadyuve a su autoevaluación. WhatsApp, adquiere así, una valoración indubitable para el trabajo colaborativo grupal, como espacio promotor del aprendizaje estudiantil desde la perspectiva constructivista, a la luz de la motivación y comunicación solidaria entre sus miembros que comparten las mismas metas en común.

\section{Conclusiones}

La coyuntura sanitaria mundial provocada por la pandemia del COVID-19, ha dado lugar a que la comunicación en grupo que se establece a través de WhatsApp, cobre significativa importancia y mayor vigencia en el ámbito educativo universitario mediante el desarrollo del aprendizaje colaborativo, lo cual demanda a estudiantes y docentes, un mayor grado de sensibilización y compromiso mutuo, para aprovechar el potencial comunicativo que esta herramienta tecnológica puede brindar en el proceso de enseñanza-aprendizaje. Entre las conclusiones arribadas en el presente análisis documental, se resalta en primer lugar que, el uso de WhatsApp como medio para el aprendizaje colaborativo, implica una apuesta solidaria de actitudes favorables a la cooperación mutua entre los participantes de un mismo enclave comunicativo (estudiantes y docentes que se comunican en un grupo de WhatsApp). Un punto a favor en esta relación comunicativa, está determinada por el carácter de flexibilidad e informalidad que posee en las conversaciones grupales producidas entre los estudiantes, donde el lenguaje no resulta una materia revisable y otorga una mayor libertad en la forma como se desea intercambiar los mensajes en grupo, sin embargo, puede denotar un aspecto en contra, si se considera que la construcción gramatical de las oraciones y respeto a las normas de ortografía, puedan generar consecuencias adversas en la comunicación de acuerdo a lo señalado por Kisel, (2019). Cabe destacar que, el distanciamiento social durante la pandemia, obliga que las comunicaciones interpersonales se caractericen por su claridad, fluidez y menor grado de aspectos subjetivos, puesto que la comunicación que se establece por medio de estas herramientas tec- 
nológicas, podría afectar la intención del mensaje que busca dirigirse. En segundo lugar, que el uso de WhatsApp en la comunicación establecida por un grupo de estudiantes, se sitúa en el contexto de la teoría de los usos y gratificaciones de Katz, Lundberg, Hulten, Mc Quail, si asumimos que el aprendizaje colaborativo es el resultado de las gratificaciones obtenidas, a partir del consumo informativo que registran los estudiantes universitarios al seleccionar y elegir este medio para usarlo y satisfacer sus necesidades educativas. Tercero, el aprendizaje colaborativo que vaya a lograrse desde las relaciones comunicativas en un grupo de WhatsApp, demanda por la misma coyuntura, una mayor empatía y resiliencia de cada docente para acercarse a sus estudiantes. Recordemos que la educación a distancia, debe caracterizarse por establecer un contacto comunicativo permanente y motivador, que propicie un aprendizaje colaborativo basado en la construcción holística de conocimientos, que parten desde la enseñanza y apoyado en el uso de las herramientas tecnológicas. En este sentido, se desprende una cuarta conclusión que afianza la importancia de la tutoría en las conversaciones grupales por WhatsApp, para acercar la clase al estudiante e identificar sus principales dificultades, al mejorar su motivación (Hassan \& Ahmed, 2018), participación (Fuentes, et, al, 2017) y número de interacciones (Lizcano-Dallos, Barbosa-Chacón y Villamizar-Escobar, 2019) que coadyuve a mejorar su proceso de enseñanza-aprendizaje en medio de esta coyuntura (Hodges, et, al, 2020). Finalmente, indicar que la COVID-19, nos deja así, la lección de encontrarnos preparados al cambio; toda vez que en el contexto educativo y social en su conjunto, ha quedado demostrado que el uso y manejo de las tecnologías de comunicación como WhatsApp para el aprendizaje colaborativo, se tornan cada vez más indispensables y de conocimiento obligatorio entre las personas; inclusive en aquellas que hasta hace poco, veían con escepticismo, que se pueda desarrollar educación a distancia con experiencias colaborativas grupales importantes, fundamentadas desde las teorías de usos y gratificaciones y complementariedad del canal. Al tratarse de un hecho coyuntural que ha afectado el mundo en 2020, se recomienda desarrollar estudios aplicados sobre las distintas experiencias grupales de conversación en WhatsApp, registradas entre estu- diantes y docentes, a la luz de la naturaleza de cada materia, perfil pedagógico y tutorial y formas de desarrollo de los contactos comunicativos, con el fin de vislumbrar como los niveles de eficacia del aprendizaje colaborativo desde el uso de sistemas de mensajería instantánea como WhastApp, en medio de la pandemia del COVID-19.

\section{REFERENCIAS BIBLIOGRÁFICAS}

Alsina, M. R. (2018). Teorías de la comunicación: ámbitos, métodos y perspectivas, v. 11, Aldea Global. Universitat Autónoma de Barcelona. Recuperado de https://books.google. com.pe/books?id=Oep6DwAAQBAJ\&lpg=PA1\&hl=es\&p$\mathrm{g}=\mathrm{PA} 1 \# \mathrm{v}=$ onepage $\& \mathrm{q} \& \mathrm{f}=$ false

Bangisisi Z, Mathews N. (2020) Development of a Quantitative Instrument to Measure Mobile Collaborative Learning (MCL) Using WhatsApp: The Conceptual Steps. En. Nyembe B.Z.M., Howard G.R, Pappas I., Dwivedi Y, Mäntymäki M. (eds) Responsible Design, Implementation and Use of Information and Communication Technology. I3E 2020. Lecture Notes in Computer Science, vol 12066. Springer, Cham

https://doi.org/10.1007/978-3-030-44999-5_42

Casero-Ripollés, A. (2020). Impact of COVID-19 on the media system. Communicative and democratic consequences of news consumption during the outbreak. El profesional de la información, 29(2). https://doi.org/10.3145/epi.2020.mar.23

Castellanos, J. C., Onrubia, J. (2016). La importancia de los procesos de regulación compartida en CSCL; rasgos teóricos y empíricos para su estudio. Revista Iberoamericana De Educación, 70(1), 29-46. https://doi.org/10.35362/rie70171

Díaz Quiñones, J., Iglesias León, M., Valdés Gómez, M. (2020). La tutoría a distancia: acciones del tutor en la Estrategia de Formación Doctoral en tiempos de COVID 19. Medisur, 18(3), 478-484. Recuperado de http://www.medisur.sld.cu/index.php/medisur/article/view/4678

D'Orville, H (2020). COVID-19 causes unprecedented educational disruption: Is there a road towards a new normal?. Prospects. https://doi.org/10.1007/s11125-020-09475-0

Elhay, A.A., Hershkovitz, A. (2019) Teachers' perceptions of out-of-class communication, teacher-student relationship, and classroom environment. Educ Inf Technol 24, 385-406. https://doi.org/10.1007/s10639-018-9782-7

Fondevila-Gascón, JF, Marqués-Pascual, J, Mir-Bernal, P, Polo-López M. (2019): Usos del WhatsApp en el estudiante universitario español. Pros y contras. Revista Latina de Comunicación Social, 74, 308-324.

https://doi.org/10.4185/RLCS-2019-1332

Fuentes, V., García, M., Aranda, M., Aranda López, M. (2017). Grupos de clase, grupos de WhatsApp. Análisis de 
las dinámicas comunicativas entre estudiantes universitarios. Revista Prisma Social, (18), 144-171. Recuperado de https://revistaprismasocial.es/article/view/1448

Gómez Del Castillo, M. (2017). Utilización de WhatsApp para la Comunicación en Titulados Superiores. REICE Revista Iberoamericana sobre Calidad, Eficacia y Cambio en Educación, 15 (4), 51-65. https://doi.org/10.15366/reice2017.15.4.003

Hassan, A., \& Ahmed, S. S. (2018). The impact of WhatsApp on learners' achievement: A case study of English language majors at King Khalid University. International Journal of English Language Education, 6(2), 69-81.

https://doi.org/10.5296/ijele.v6i2.13743

Hodges, C., Moore, S., Lockee, B., Trust, T., Bond, A. (2020). The Difference Between Emergency Remote Teaching and Online Learning. Educause Review. https://er.educause.edu/ articles/2020/3/the-difference-between-emergency-remote-teaching-and-online-learning

Igartua, J, Ortega-Mohedano, F., Arcila-Calderón, C. (2020). The uses of communication in the time of coronavirus. A cross-cultural study. El profesional de la información, 29 (3), e290318. https://doi.org/10.3145/epi.2020.may.18

Kizel, A. (2019) I-Thou dialogical encounters in adolescents' WhatsApp virtual communities. AI \& Soc 34, 19-27. https://doi.org/10.1007/s00146-017-0692-9

Jain J., Eddy Luaran J.., Rahman N..A. (2016) Learning Beyond the Walls: The Role of WhatsApp Groups. In: Luaran J., Sardi J., Aziz A., Alias N. (eds) Envisioning the Future of Online Learning. Springer, Singapore. https://doi. org/10.1007/978-981-10-0954-9_40

Karapanos, E., Teixeira, P., Gouveia, R. (2016). Need fulfillment and experiences on social media: a case on Facebook and WhatsApp. Computers in human behavior, 55, 888897. https://doi.org/10.1016/j.chb.2015.10.015

Lacanna, G. A. (2018). No des más vueltas. Un estudio del uso de la indireccionalidad en la realización de invitaciones y propuestas en interacciones por WhatsApp. Calidoscópio, 16(2), 225-236. https://doi.org/10.4013/cld.2018.162.05

Lizcano-Dallos, A., Barbosa-Chacón, J., Villamizar-Escobar, J. (2019). Aprendizaje colaborativo con apoyo en TIC: concepto, metodología y recursos. Magis, Revista Internacional de Investigación en Educación, 12(24), 5-24. https://doi. org/10.11144/Javeriana.m12-24.acat

López-Cantos, F. (2017). WhatsApp. Hábitos de uso y construcción de identidad visual con mensajería móvil, Icono 14, volumen 15 (2), 70-91. https://doi.org/10.7195/ri14.v15i2.996

Martínez, D. (2020). Un texto paralelo sobre el COVID-19. Question/Cuestión,

https://doi.org/10.24215/16696581e374

Montenegro D, Hermenegildo E. (2018) Características del uso de emojis en la comunicación por el chat de WhatsApp. Rev. Hamut'ay. Vol. 5 (1). https://doi.org/10.21503/hamu. v5i1.1519
Moreno, A; Fuentes-Lara, C; Navarro, C. (2020). COVID-19 communication management in Spain: Exploring the effect of information-seeking behavior and message reception in public's evaluation. El profesional de la información, 29(4), https://doi.org/10.3145/epi.2020.jul.02

Rivera-Rogel D., Yaguache, J., Velásquez A., Paladines, F. (2019) Social Networks as a New University Venue. In: Túñez-López M., Martínez-Fernández VA., López-García X., Rúas-Araújo X., Campos-Freire F. (eds) Communication: Innovation \& Quality. Studies in Systems, Decision and Control, 154. Springer. https://doi.org/10.1007/978-3319-91860-0_30

Rubio Romero, J., Perlado Lamo de Espinosa, M. (2015). El fenómeno WhatsApp en el contexto de la comunicación personal: una aproximación a través de los jóvenes universitarios. Revista ICONO14 Revista científica de comunicación y tecnologías emergentes, 13(2), 73-94. https://doi. org/10.7195/ri14.v13i2.818

Ruiz, E. (2020). La práctica docente universitaria en ambientes de educación a distancia. Tensiones y experiencias de cambio. En H. Casanova Cardiel (Coord.), Educación y pandemia: una visión académica (pp. 109-113). Ciudad de México: Universidad Nacional Autónoma de México, Instituto de Investigaciones sobre la Universidad y la Educación. Recuperado de http://132.248.192.241:8080/jspui/ bitstream/IISUE_UNAM/545/1/RuizLarraguivelE_2020_ La_practica_docente.pdf

Sánchez Mendiola, M, Martínez Hernández, A, Torres Carrasco, R. Servín, M, Hernández Romo, A., Benavides Lara, M., Jaimes Vergara, C. y Rendón Cazales, V. (2020). Retos educativos durante la pandemia de COVID-19: una encuesta a profesores de la UNAM. Revista Digital Universitaria (RDU), 21(3), https://doi.org/10.22201/codeic.16076079e.2020.v21n3.a12

Sánchez-Duarte, J., Magallón Rosa, R. (2020). Infodemia y COVID-19. Evolución y viralización de informaciones falsas en España. Revista española de comunicación en salud, 0, 31-41. https://doi.org/10.20318/recs.2020.5417

Sanz, I., Sáinz González, J., Capilla, A. (2020). Efectos de la Crisis del Coronavirus en la Educación Superior. Organización de Estados Iberoamericanos para la Educación, la Ciencia y la Cultura (OEI). Recuperado de https://oei.org. br/arquivos/informe-COVID-19d.pdf

Scribano, A. (2017). Miradas cotidianas. El uso de WhatsApp como experiencia de investigación social. Revista Latinoamericana de Metodología de la Investigación Social, 13 (7), 08-22. http://www.relmis.com.ar/ojs/index.php/relmis/ article/view/207

Suárez, B. (2017) El WhatsApp como herramienta de apoyo a la tutoría. REDU. Revista de Docencia Universitaria, 15 (2), 193-210, https://doi.org/10.4995/redu.2017.6941

Suárez, B. (2018). WhatsApp: su uso educativo, ventajas y desventajas. Revista de Investigación en Educación, 16. (2) 121-135. Recuperado de. http://reined.webs4.uvigo.es/in- 
dex.php/reined/article/view/342

Tang, Y, Hew, K. (2020) Does mobile instant messaging facilitate social presence in online communication? A two-stage study of higher education students. Int J Educ Technol High Educ 17, 15. https://doi.org/10.1186/s41239-020-00188-0

Urien, B., Erro-Garcés, A., Osca, A. (2019). WhatsApp usefulness as a communication tool in an educational context. Education and Information Technologies, 24(4), 25852602. https://doi.org/10.1007/s10639-019-09876-5

Vela Delfa, C, Cantamutto, L. (2016). De participante a observador: el método etnográfico en el análisis de las interacciones digitales de WhatsApp. Repositorio Institucional UNS, Argentina. http://repositoriodigital.uns.edu.ar/handle/123456789/4054

Vera Carrasco, O. (2009). Cómo escribir artículos de revisión. Revista Médica La Paz, 15(1), 63-69. Recuperado de http://www.scielo.org.bo/scielo.php?script=sci_arttext\&pi$\mathrm{d}=$ S1726-89582009000100010\&lng=es\&tlng=es

Vilela, M. J. V., Urbano, E. R.,Díaz, V. M. (2015). Diseño y validación de un cuestionario para valorar WhatsApp en la regulación de trabajo en grupo. Revista científica electrónica de Educación y Comunicación en la Sociedad del Conocimiento, 15(2). Recuperado de http://www.eticanet.org/revista/index.php/eticanet/article/view/78/72

Vilches, M. J., Reche, E. (2019). Limitaciones de WhatsApp para la realización de actividades colaborativas en la universidad. RIED. Revista Iberoamericana de Educación a Distancia, 22(2), 57-77. https://doi.org/10.5944/ried.22.2.23741 